\title{
Caracterización del campo de acción laboral de los graduados de la Facultad de Ciencias Contables, Administrativas y Económicas de la UNP
}

\author{
Diana Azucena Rolón Ramírez \\ azucenarolon17@gmail.com \\ Ramón Plutarco Guillén Segobia \\ plutarkoz@hotmail.com \\ Laura Belén Torres Montañez \\ lauritatm92@hotmail.com \\ Manuel Alejandro Bourdier Fernández \\ manuale1987@hotmail.com \\ Facultad de Ciencias, Tecnologías y Artes \\ Universidad Nacional de Pilar
}

\section{RESUMEN}

Este trabajo describe el campo de acción en el que se desempeñan los graduados de la Facultad de Ciencias Contables, Administrativas y Económicas de la UNP. La justificación de este abordaje coincide con Medina García \& Rojas Asprilla (2014) en conocer la situación por la que atraviesan los profesionales que actualmente laboran, o que por algún motivo están desempleados o se desempeñan en oficios diferentes a la Contaduría. Este propósito fue realizado mediante una metodología con enfoque cuantitativo, de tipo descriptivo, con un diseño observacional, transversal y prospectivo. Como técnica de recolección de datos se valió de un censo utilizando un cuestionario aplicado a los graduados, comprendido entre los periodos 2014 al 2018. Como resultado se detectó que el 95\% de los encuestados están laborando actualmente, la mayor parte no se desempeña como contador propiamente, tan solo 6 profesionales se dedican a la Contabilidad de manera independiente como actividad principal, sin embargo, los encuestados restantes laboran en áreas relacionadas al ámbito contable. Por último, la mayor parte de los graduados si ejercen la contabilidad, pero como una actividad secundaria, es decir, lo realizan como otra fuente adicional.

Palabras clave: caracterización, campo de acción laboral, contador público, graduados, ejercicio profesional. 


\title{
Characterization of the labor action field of graduates of the Faculty of Accounting, Administrative and Economic Sciences of the UNP
}

\begin{abstract}
This work describes the field of action in which graduates of the Faculty of Accounting, Administrative and Economic Sciences of the UNP perform. The rationale for this approach coincides with Medina García \& Rojas Asprilla (2014) in to know about the situation that the professionals actually working are going through, or that for some reason are unemployed or work in occupation other than Accounting. This purpose was accomplished through a methodology with a quantitative approach, of a descriptive type, with an observational, transversal and prospective design. As data collection technique, employed a census using a questionnaire applied to graduates, between the periods 2014 to 2018. About results, it was detected that $95 \%$ of respondents are currently working, most of them do not labored like an accountant properly, and only 6 professionals are dedicated to Accounting independently as the principal activity, however, the remaining respondents work in areas related to the accounting field. Finally, most graduates do practice accounting, but like a secondary activity, that to say, they do it as another additional source.
\end{abstract}

Keywords: characterization, labor action field, public accountant, graduates, professional practice.

Artículo recibido: 02. ene. 2020

Aceptado para publicación: 10. ene. 2020

Correspondencia: azucenarolon17@gmail.com

Conflictos de Interés: Ninguna que declarar 


\section{INTRODUCCIÓN}

Este trabajo consiste en caracterizar el campo de acción laboral de los contadores públicos graduados de la Facultad de Ciencias Contables, Administrativas y Económicas de la Universidad Nacional de Pilar. Según la afirmación de Damian (2014), el mercado laboral requiere que los graduados posean una formación polivalente y flexible, es decir, que este tenga la capacidad o habilidad de desenvolverse en diferentes áreas laborales. Por tal motivo, se formula la siguiente interrogante ¿se ven los graduados en la necesidad de incurrir en otras fuentes laborales las cuales pueden o no tener relación de su profesión?

De modo a caracterizar a los graduados dentro del mercado de trabajo es necesario definir la inserción laboral o inserción profesional dentro del mismo. Para Barrón (2005) "la inserción laboral es definida como la consecución y desempeño de un empleo dentro de un área y sector acorde con la formación recibida" (como se citó en Damián, 2014, pág.14). Para enfatizar mejor este concepto, se entiende a la Inserción Laboral como el periodo en que el graduado pasa desde la culminación del plan de estudios y obtención del título, hasta conseguir un trabajo estable, y todas las experiencias en que éste incurre durante este lapso, proporcionando una base para la reflexión de la interacción entre la Educación Superior y el empleo como concluye Damián, (2014)

Por otro lado, para Sánchez Upegüi (2010) "Desde una perspectiva investigativa la caracterización es una fase descriptiva con fines de identificación, entre otros aspectos, de los componentes, acontecimientos (cronología e hitos), actores, procesos y contexto de una experiencia, un hecho o un proceso". (como se cito en Rojas Asprilla \& Medina García, 2014, pág. 21).

En cambio, para Bonilla Castro y Otros, la caracterización es un tipo de descripción que puede recurrir a datos con el fin de profundizar el conocimiento sobre algo. Para caracterizar ese algo previamente se deben identificar y organizar los datos, por ejemplo, de encuestas; y a partir de ellos, describir (caracterizar) de una forma estructurada; y posteriormente, establecer su significado. (como se cito en Rojas Asprilla \& Medina García, 2014, pág. 21).

Otros conceptos relevantes en esta investigación son las de los Egresados y Graduados, la distinción radica en que egresado es aquel que ha finalizado efectivamente el plan de estudios; y por otro lado graduado es quien ya ha obtenido el título universitario, en base a lo mencionado por Jaramillo, et al (2006). 
Para Romero Fernández y Álvarez Gavilanes (2015), es indiscutible que los constantes y rápidos cambios que se producen en el mercado laboral tienen una gran influencia en la empleabilidad de los graduados, estos tienen que aprender a ser más flexibles, estar preparados para aprender, desaprender y aprender nuevamente, potenciar habilidades comunicativas y de relaciones sociales dentro del sector empresarial donde laboran. (como se cito en Gette y Otros, 2018, pág. 88). Asimismo, García y Rincón (2016), afirman que es indispensable el ejercicio contable para el sector empresarial, y que estos deben estar al corriente de avances tecnológicos, actualizaciones constantes y los potenciales cambios legales (como se cito en Molina Gutiérrez, et al, 2018, pág. 166).

Teniendo en cuenta lo mencionado, según el Instituto Tecnológico de Sonora (2005) el contador, es un profesional cuya función es la de proporcionar información financiera a las entidades económicas, mediante la creación de servicios de consultoría, auditoría e impuestos, y por medio de la reflexión, análisis y síntesis, emitir juicios para la toma de decisiones, contribuyendo al desarrollo de las organizaciones y de la sociedad. (Celaya Figueroa, López Parra, \& Aceves López, 2008, pág. 45).

En la República del Paraguay, el Contador Público es un profesional universitario, formado con sólidos conocimientos científicos y técnicos, sumados a una buena formación integral, un experto en contabilidad, en auditoría, en áreas financieras y fiscal, así como en planeación, emprendimientos para la creación de nuevos negocios, análisis, crítica, interpretación e investigación de las finanzas, y un buen investigador en las áreas de contabilidad y auditoría. Tendrá en cuenta las Normas Internacionales de Información Financiera, las NIIF completas como las NIIF para Pymes y las del sector público. Así como las Normas Internacionales de Auditoría para todos los sectores y las Normas Contables Nacionales. (Agencia Nacional de Evaluación y Acreditación, 2013)

La relación entre los graduados y las Universidades es de vital importancia, por lo cual coincidimos con los siguientes autores cuando expresan; López et al (2010), sostienen que el vínculo por parte de las instituciones educativas y sus graduados es de suma importancia ya que a partir de ésta permite mejorar los procesos de enseñanza académica, mediante el contraste y la retroalimentación de la realidad de los profesionales y la enseñanza superior (como se citó en Gómez Cano, et all, 2016, pág. 31).

Al respecto Marulanda Galvis, et al (2010), infieren en que hay una necesidad de un análisis riguroso de las instituciones para con sus graduados, en tal sentido a ser capaces de satisfacer las demandas de una sociedad cada vez más exigente. 
Ospino (2014), refiere que los estudios a graduados buscan contribuir a la explicación del desempeño profesional, posibilitando realizar un seguimiento continuo que permita no sólo conocer la situación de los mismos en un momento determinado, sino comparar su evolución de acuerdo con su perfil académico y la situación del mercado laboral. ( como se citó en Mercado Caruso, y Otros, 2015, pág. 21).

En base a lo dicho anteriormente es necesario contar con una investigación de este tipo de modo a evidenciar la situación laboral en el que se desempeñan los graduados, es decir, están insertos dentro del mercado, ejercen o no la profesión contable, trabajan por cuenta propia, se encuentran en instituciones públicas o privadas, si los profesionales se encuentran identificados con el perfil de egreso de la Facultad, siguen otros estudios superiores y si están satisfechos con su remuneración salarial. Por otra parte, también se podrán obtener datos para cuantificarlos y tener registros en aspectos como edad, sexo y año de egreso. Por tal motivo esta investigación es de utilidad para la misma universidad de modo a proveer información sobre la inserción de sus graduados dentro del mercado laboral.

Algunos motivos que se perciben dentro del mercado laboral, por el cual los graduados no ejercen la profesión son las siguientes: por la baja remuneración, por la falta de experiencia, financiamiento, motivación, por la infravaloración de su labor, y algunos porque la formación que recibieron no llega a cumplir sus expectativas para desempeñarse dentro del sector.

Uno de los principales antecedentes tomados para esta investigación corresponde al trabajo realizado por Rojas Asprilla \& Medina García, (2014, pág. 13) cuyo trabajo tiene por objetivo "conocer la situación por la que atraviesan los profesionales que actualmente laboran, o que por algún motivo están desempleados o que se encuentran desempeñando oficios en áreas diferentes a la Contaduría". Asimismo, Mercado Caruso et al (2015) hacen mención que la profesión contable es una de las más dinámicas, en el diagnostico laboral del graduado del programa de Contaduría Pública.

El Proyecto educativo de la carrera de la Facultad de Ciencias Contables, Administrativas y Económicas menciona los posibles campos laborales en los cuales pueden desempeñarse los contadores Públicos, la misma expresa lo siguiente:

El egresado de la carrera podrá desempeñarse como asesor contable, financiero, tributario e impositivo, y auditor de entidades públicas y privadas, entidades descentralizadas, organismos departamentales y municipales, bancos y otras entidades del sector financiero; empresas comerciales, industriales y de servicios, 
empresas ganaderas. Será capaz de formular, interpretar y analizar estados financieros, elaborar y actualizar inventarios, de acuerdo a las normas de la profesión, y demás normas nacionales e internacionales de información financiera. (Facultad de Ciencias Contables, 2018).

Por otra parte, el perfil de egreso de la Carrera expresa lo siguiente:

El egresado de la Carrera de Contaduría Pública Nacional posee sólidos conocimientos científicos y técnicos, además de una formación integral que le permiten elaborar información financiera de empresas, entidades públicas y organismos no gubernamentales para la toma de decisiones; adaptándose también a los escenarios económicos, financieros, empresariales y sociales. Además, tiene capacidad para emprender, asesorar, gerenciar su propio negocio y anticiparse a los cambios del entorno a nivel nacional, regional e internacional. (Facultad de Ciencias Contables, 2018).

\section{METODOLOGÍA}

Este propósito fue realizado mediante una metodología con enfoque cuantitativo, de tipo descriptivo, con un diseño observacional, transversal y prospectivo. Como técnica de recolección de datos se valió de un censo utilizando un instrumento documental, en este caso el cuestionario aplicado a los graduados en Contaduría Pública de la Universidad Nacional de Pilar comprendido entre los periodos 2014 al 2018. Dicho cuestionario es el utilizado por Leiva Medina García y Dissan Lorena Rojas Asprilla en su trabajo de grado denominado Caracterización del campo de acción laboral de los contadores públicos graduados de la Universidad Del Valle sede Pacifico del año 2005 al 2010, la cual fue adaptado a las necesidades de esta investigación.

La población de estudio lo conformaron graduados de la carrera en: Licenciatura en Ciencias Contables; Auditoria y Contaduría Pública Nacional; y, Contaduría Pública Nacional, desde el año 2014 al 2018, totalizando 75 graduados, según listado proveído por la Secretaría General de la Facultad de Ciencias Contables, Administrativas y Económicas de la Universidad Nacional de Pilar.

Cabe mencionar que se excluyeron a los graduados de otras filiales de la Facultad de Ciencias Contables, Administrativas y Económicas que totalizan 12 personas, así como tampoco se incluyen a 7 graduados de la sede Pilar que no residen dentro de la misma ciudad, debido al difícil acceso a éstos. 
Se tuvo en cuenta consideraciones éticas como el consentimiento informado, el tratamiento anónimo de los datos, el respeto a los valores y creencias de los sujetos participantes, así como la libertad de opinión, etc.

\section{RESULTADOS Y DISCUSIÓN.}

A continuación, se presentan los resultados más relevantes que se obtuvo a partir de esta investigación distribuida en cuatro apartados: características generales, identificación laboral de los graduados, identificación con el perfil de egreso y satisfacción salarial.

En un inicio se realiza una descripción general de los graduados encuestados, por medio del cual se detectó que, del total, 19 son hombres y 56 son mujeres, lo que representa un $25 \%$ y $75 \%$ respectivamente.

Estos resultados coinciden con Himitian (2013), quien menciona "que las mujeres lideran la participación en el total de títulos otorgados, especialmente en el nivel universitario.”, por tanto, se refleja el mayor interés y la dedicación por parte de las mujeres para obtener el título de grado y llegar a ser profesionales en el ramo de la contabilidad (como se cito en Gómez Cano et al, 2016, pág. 32).

Cabe mencionar, que el rango de edad de los graduados oscila entre los 26 a 29 años que representa un $39 \%$ de la población de estudio.

Entre los periodos estudiados, la mayor cantidad de graduados tienen el título de Auditor y contador Público Nacional con un $75 \%$, seguido por un $20 \%$ de graduados en Contaduría Pública Nacional y 5\% Licenciados en Ciencias Contables. Esta última (Licenciatura en Ciencias Contables) fue la primera carrera habilitada por la Facultad de Ciencias Contables, Administrativas y Económicas, por tanto, los resultados expuestos representan a aquellos que recién obtuvieron su título de grado durante el periodo 2014 a 2018. La segunda es la de Auditoria y Contaduría Pública Nacional, es la de mayor representatividad, este inicio desde el año 2005 y culmino en el 2009; y, la última es la que corresponde a Contador Público Nacional que empezó desde el 2010 hasta el 2017. Además, se destaca que la mayor cantidad de graduados obtuvieron su título de grado en el año 2018 que representa el $46 \%$ del total. 
Gráfico 1: Título de Grado

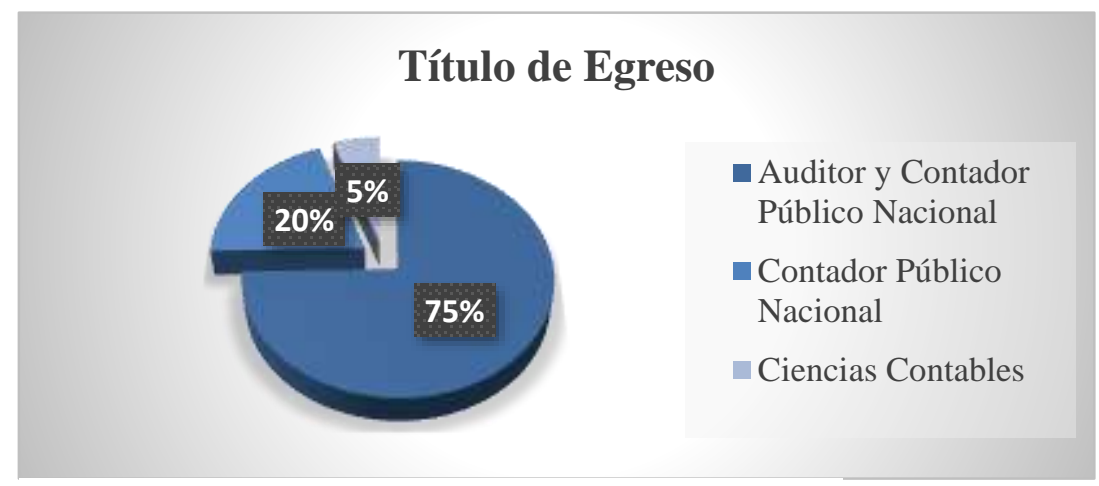

Fuente: Elaboración Propia.

Dando paso al siguiente apartado, situación laboral del contador público, el 95\% manifestó estar laborando actualmente y solo el 5\% no lo está. Por lo tanto, se evidencia la buena aceptación de los graduados en el campo laboral de la ciudad de Pilar.

Atendiendo el sector en que se desempeñan, cabe mencionar que el $49 \%$ trabaja en el sector privado, el $31 \%$ en el sector público, tan solo el $8 \%$ se dedica al ejercicio de la profesión en forma independiente, el $7 \%$ trabaja en otros rubros diferentes a la contabilidad de manera independiente y el 5\% manifestó no estar trabajando. En discrepancia con Ortega (2012) que en su estudio en Colombia detecto que el principal empleador es el Estado, ya sea en forma directa o indirecta. (como se cito en Gómez Sánchez, Oviedo Marín, \& Martínez López, 2011, pág. 95).

Algunos de los motivos manifestados por los graduados por lo cual no se dedican al ejercicio de la profesión por cuenta propia son las siguientes: la falta de experiencia, de motivación, de financiamiento, de conocimiento y por la baja remuneración en los trabajos contables.

En cuanto a las actividades en que desempeñan el $44 \%$ mencionaron que poseen más de una actividad laboral, el 51\% no lo posee, y tan solo el 5\% no trabaja. El ejercicio de la actividad profesional en el área de Contabilidad a nivel país de acuerdo a la DGEEC del año 2002, refleja que la población ocupada está compuesta por el $86,7 \%$; desocupados por $2,4 \%$; inactivos por 10,8\% y los no informado por un 0,1\% (Ramírez Caballero \& Brizuela Bordón, 2002, pág. 51).

Con respecto a su actividad principal, se observa que 27 profesionales están insertos en el área de Administración, 15 en área de Contabilidad y 11 en Finanzas. Como actividad secundaria 21 profesionales se dedican a la profesión contable, 7 en otros campos laborales diferentes al área de contabilidad, 3 en el ámbito de Docencia y, por último, 2 en Administración. Mercado et al. (2015) señala que la diversidad de cargos que ocupa el contador público al iniciar su vida laboral se debe a la falta de oportunidades en el sector productivo. Así mismo Arquero et al. (2007) hace mención en que los contadores se desenvuelven en otras áreas de la empresa fuera de lo contable. 
Gráfico 2: Actividad Principal

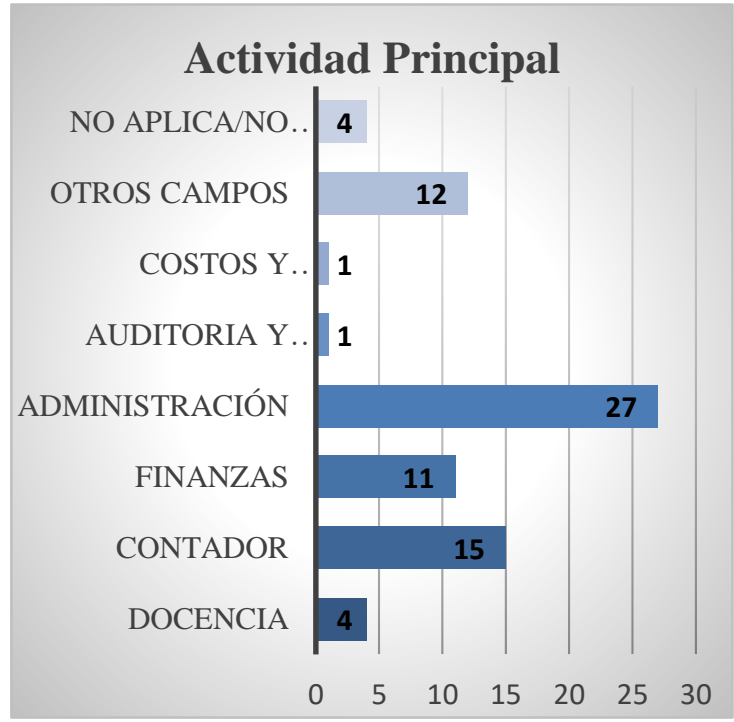

Fuente: Elaboración Propia.
Gráfico 3: Actividad Secundaria

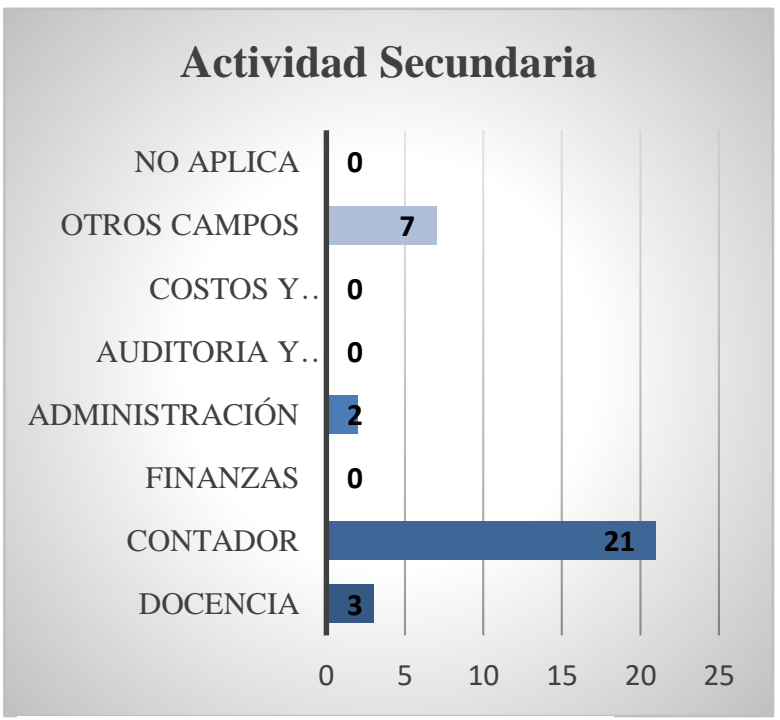

Fuente: Elaboración Propia.

En cuanto el tercer aspecto estudiado, el cual refiere al apartado Institucional, el 73\% se siente identificado con el Perfil de Egreso, en cambio el 16\% está en desacuerdo y finalmente un 11\% se abstuvo de responder. Por tanto, los resultados reflejan que los graduados se sienten identificados con todos los requisitos establecidos en el perfil de egreso. Es decir, los mismos poseen; sólidos conocimientos científicos y técnicos, además de una formación integral que le permiten elaborar información financiera de empresas, entidades públicas y organismos no gubernamentales para la toma de decisiones; adaptándose también a los escenarios económicos, financieros, empresariales y sociales. Además, tiene capacidad para emprender, asesorar, gerenciar su propio negocio y anticiparse a los cambios del entorno a nivel nacional, regional e internacional (Facultad de Ciencias Contables, 2018)

Gráfico 4: Se siente identificado con el perfil de egreso

Se siente identificado con el Perfil de Egreso

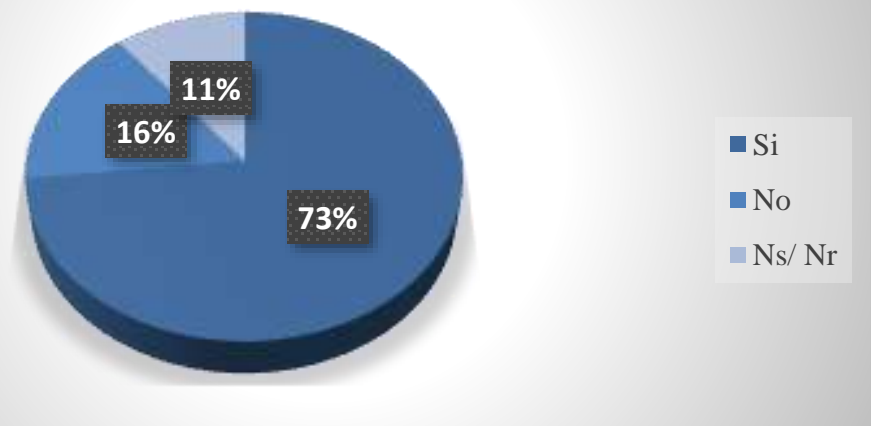

Fuente: Elaboración Propia. 
En cuanto a la preparación recibida en la Facultad, el $72 \%$ manifestó que la formación está de acuerdo al campo laboral en que se desempeñan.

El último apartado, Satisfacción Salarial, en su mayoría un $71 \%$ corresponde a graduados que están insertos en otros ámbitos laborales distintos al área contable. Sin embargo, el 16\% que si se desempeña en el rubro contable considera que su salario no va acorde con la labor, ya que los mismos no alcanzan el mínimo vigente, y tan solo el 9\% si lo percibe. También cabe comparar con lo mencionado por Barrios y Corchuelo (2015) (citado en Gómez Cano et al. 2016), que concluye que existe una marcada insatisfacción con la remuneración percibida (pág. 33).

También, el estudio realizado por Díaz Santa \& Restrepo Castrillón, (2013) los profesionales encuestados opinan que en Colombia el ejercicio de la contaduría pública no está bien remunerado, demostrando que a nivel nacional resulta difícil establecer un honorario base para los Contadores (como se cito enVarón García , 2016, pág. 4).

\section{Gráfico 5: Salario como Contador}

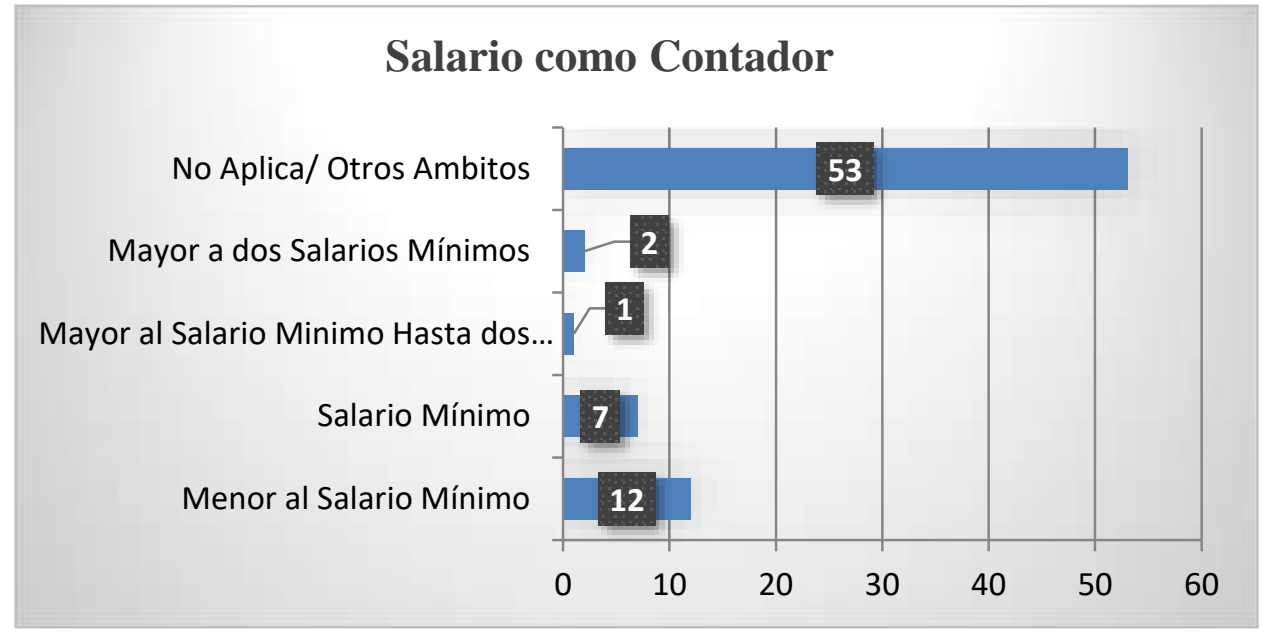

Fuente: Elaboración Propia.

En cuanto al salario en otros ámbitos ajenos a la Contabilidad los resultados arrojados demuestran que 2 profesionales perciben mayor a dos salarios, 7 el mínimo vigente. Por lo cual queda en evidencia que el ejercicio de la contabilidad pasa a ser un complemento de la actividad principal de los graduados, ya que las remuneraciones son mayores en otros ámbitos del mercado laboral. 


\section{CONCLUSIÓN}

El objetivo de esta investigación es describir el campo de acción laboral de los graduados de la Facultad de Ciencias Contables, Administrativas y Económicas, para llegar al cumplimiento del mismo, se tuvo acceso a 75 graduados quienes proporcionaron informaciones pertinentes a través de la aplicación del instrumento de recolección de datos.

Mediante ésta, se constató que la aceptación de los graduados dentro de la ciudad de Pilar es bastante alta, ya que el 95\% se encuentran trabajando actualmente. Uno de los resultados más resaltante fue que, tan solo 6 profesionales se dedican a la Contabilidad propiamente, y de manera independiente, sin embargo, se evidencia que 60 de ellos están insertos en instituciones del sector público y privado, en su mayoría en carácter de dependencia en áreas Administrativas y Financieras ejerciendo labores altamente relacionadas al ámbito contable tomando como referencia su Actividad Principal.

En base a los resultados obtenidos teniendo al indicador de poseer más de una actividad laboral, se infiere que la mayor parte de los graduados ejercen la contabilidad, pero lo hacen como una actividad secundaria, es decir, lo realizan como otra fuente adicional para aumentar sus ingresos.

Asimismo, es relevante resaltar la necesidad manifestada por los graduados, de poner énfasis en; ejercicios prácticos actualizados durante el proceso de enseñanza y aprendizaje, mayor seguimiento a los graduados, priorizar capacitaciones, fomentar la habilitación de más carreras de postgrado relacionados al área contable.

Por otro lado, la mayoría declaró que no ejercen la contabilidad como actividad principal debido a la falta de regulación e interés por unificar los honorarios, lo que conlleva a una desvalorización de la profesión contable, muchos de los que ejercen la contabilidad no realizan un análisis previo del tiempo invertido y los costos incurridos para la presentación de una oferta al cliente.

En relación al perfil de egreso la investigación arrojó resultados favorables, ya que la mayor parte de los encuestados afirmó sentirse identificado con el perfil de egreso correspondiente a la carrera de contabilidad de la Facultad.

Con la elaboración de este artículo científico podemos decir, que llegamos a cumplir con el objetivo de la investigación, no obstante, los resultados presentados pueden servir como punto de partida para futuras investigaciones y de esta forma profundizar sobre temas específicos relacionados a esta línea de investigación.

A modo de recomendación, para otras investigaciones podemos mencionar las siguientes interrogantes: ¿Qué motivo impulsa a los encuestados a laborar en campos diferentes a la 
contaduría pública? y ¿Cuáles son las razones por las que no existe aún una asociación de contadores en Pilar?

En coincidencia con Gette et al (2018), el seguimiento de graduados, debería ser considerado un aspecto estratégico en la definición de las políticas educativas de una universidad y no debiera quedar librado a la voluntad o diligencia de equipos de investigadores o programas de actividades circunstanciales. Más bien, debería estar previsto institucionalmente en su estructura funcional de manera que se realice sistemáticamente y en forma continua. (pág. 97).

\section{REFERENCIAS}

Mercado Caruso, N., Rodriguez Quezada, N., \& Crissien Borrero, E. (2015). DIAGNÓSTICO LABORAL DEL GRADUADO DEL PROGRAMA DE CONTADURIA PÚBLICA. Económicas CUC, 19-21.

Rojas Asprilla, D. L., \& Medina García, L. (2014). CARACTERIZACION DEL CAMPO DE ACCION LABORAL DE LOS CONTADORES PUBLICOS EGRESADOS DE LA UNIVERSIDAD DEL VALLE SEDE PACIFICO DEL AÑO 2005 AL 2010. 1-67.

Agencia Nacional de Evaluación y Acreditación, d. (septiembre de 2013). Modelo Nacional de Evaluación y Acreditación, de la Educación Superior. Recuperado el 16 de Julio de 2019, de Criterios de Calidad para la Carrera de Contaduría Pública: www.ANEAES.COM

Celaya Figueroa, R., López Parra, M. E., \& Aceves López, J. N. (2008). Ética docente del contador público: un análisis comparativo internacional. Cuad. Contab., 41-56.

Damián, J. (2014). Identidad profesional, reconocimiento social e inserción laboral del universitario con formación híbrida. Propósitos y Representaciones, 9 - 43.

Facultad de Ciencias Contables, A. y. (2018). Proyecto Educativo de la Carrera. Obtenido de www.contablesunp.edu.py

Gette, M. Á., Pordomingo, E., Rodriguez, R., \& Antonietti, L. (2018). INSERCIoN LABoRAL Y TRAYECTORIA PRoFESIONAL DE LOS CONTADoRES PUBLICoS. PERSPECTIVAS de las Ciencias Económicas y Jurídicas, 81 - 98.

Gómez Cano, C. A., Sánchez Castillo, V., Fajardo, M. Y., Trucco, G. G., \& Cifuentes Garzón, D. (2016). CARACTERIZACIÓN DE LOS GRADUADOS DEL PROGRAMA DE 
CONTADURÍA PÚBLICA DE LA UNIVERSIDAD DE LA AMAZONIA 2013-2015. FACE Facultad de Ciencias Económicas y Empresariales, 29-37.

Gómez Sánchez, D., Oviedo Marín, R., \& Martínez López, E. Í. (2011). Factores que influyen en el rendimiento académico del estudiante universitario. Educación y Humanidades, 90-97.

Jaramillo, A., Giraldo Pineda, A., \& Ortiz Correa, J. S. (2006). Estudios sobre Egresados: la Experiencia de la Universidad EAFIT. Centro de Investigaciones Económicas y Financieras, 111-124.

Marulanda Galvis, J. C., Ortiz Botero, E., Moratto Vásquez, N., \& Arcila Rojas, A. P. (2010). Caracterización de egresados de la Universidad CES en las cohortes de 2003, 2005, 2007 y momento “0”. Revista CES Psicología, 50-63.

Molina Gutiérrez, T. D., Arciniegas P., O. G., \& Pantoja Burbano, M. J. (2018). Formación Universitaria y Oportunidades Laborales. Seguimiento a los graduados en Contaduría Auditoría. Horizontes de la Ciencia, 161-174.

Ramírez Caballero, R. I., \& Brizuela Bordón, M. (2002). Caracterización sociodemográfica, perfil laboral y bienestar del profesional universitario en en Paraguay. Evidencia del Censo 2002, 40-63.

Real Academia Española. (16 de Julio de 2019). Real Academia Española. Obtenido de www.rae.es

Varón García , J. K. (2016). La competencia desleal un Problema de Ética Profesional. Universidad Militar Nueva Granada, 1-16. 